\title{
Tasa de mortalidad después de una fractura de cadera en población con bajos ingresos económicos, en un hospital de entrenamiento ortopédico
}

\section{Mortality rate after a hip fracture in a low-income population in an orthopedic training hospital}

\author{
Rivera-Zarazúa S,* Vela-Goñi O,* Villarreal-Villarreal G,* Tamez-Mata Y,* \\ Ramos-Morales T,* Vilchez-Cavazos F,* Peña-Martínez V,* Acosta-Olivo C*
}

Universidad Autónoma de Nuevo León, Hospital Universitario «Dr. José E González».

RESUMEN. Introducción: Las fracturas de cadera representan una causa importante de morbimortalidad en los adultos mayores. La mortalidad a un año posterior a una fractura de cadera incrementa entre 14 y 47\%. El objetivo de este estudio fue analizar los factores de riesgo que impactan la tasa de mortalidad posterior a una fractura de cadera en una población de bajos recursos. Material y métodos: Se analizaron de manera retrospectiva pacientes con fractura de cadera traumática en un período de cuatro años en un hospital universitario con entrenamiento ortopédico. Los datos recolectados incluyeron edad, género, índice de comorbilidad de Charlson (CCI), tiempo en días para la cirugía y duración del procedimiento quirúrgico, así como necesidad de transfusión. Se analizaron dos grupos, pacientes vivos y pacientes fallecidos. Resultados: Se evaluó un total de 96 adultos mayores con fractura de cadera. La tasa de mortalidad durante el primer año fue de $16.6 \%$, mientras que al final del seguimiento fue de $32.2 \%$. El grupo de sobrevivientes presentó una mejor evaluación de acuerdo con el CCI con valor de $4.2 \pm 1.1$ versus $5.2 \pm 1.0$ en los pacientes fallecidos. No se encontró diferencia estadísticamente significativa en la mortalidad entre ambos grupos al comparar los días de espera de tiempo quirúrgico y la duración
ABSTRACT. Introduction: Hip fracture are an important cause of morbidity and mortality in old patients. The one-year mortality after a hip fracture increase between 14 to $47 \%$. The main objective was to analyze the risk factors associated with mortality after a hip fracture in a low-income population. Material and methods: Retrospective study of patients with traumatic hip fracture in a four-year period in an orthopedic training hospital. The data collected was age, gender, Charlson comorbidity index (CCI), delay time in days for surgery, duration in hours for surgical procedure, transfusion. Two groups were analyzed, alive patients and deceased patients. Results: A total of 96 patients with traumatic hip fracture was analyzed. Mortality rate in the first year was pf $16.6 \%$, and at the end of the follow-up was $32.2 \%$. The alive patients showed better values of CCI with a value of $4.2 \pm 1.1$ versus $5.2 \pm 1.0$ in the deceased patients. When compared delay time for surgery and duration of surgical procedure did not observe significant difference between patients alive and deceased. Conclusion: The delay time of surgery did not affect the mortality after a traumatic hip fracture in old patients with economic low-income.

\section{Nivel de evidencia: III}

\footnotetext{
* Universidad Autónoma de Nuevo León, Hospital Universitario «Dr. José E González».

Correspondencia:

Carlos Acosta-Olivo

Universidad Autónoma de Nuevo León, Facultad de Medicina y Hospital Universitario «Dr. José E González»,

Ave Madero y Gonzalitos, S/N, Mitras Centro, C.P. 64480.

E-mail:dr.carlosacosta@gmail.com
}

Recibido: 09-06-2020. Aceptado: 25-01-2021.

Citar como: Rivera-Zarazúa S, Vela-Goñi O, Villarreal-Villarreal G, Tamez-Mata Y, Ramos-Morales T, Vilchez-Cavazos F, et al. Tasa de mortalidad después de una fractura de cadera en población con bajos ingresos económicos, en un hospital de entrenamiento ortopédico. Acta Ortop Mex. 2021; 35(2): 193-196. https://dx.doi.org/10.35366/101865

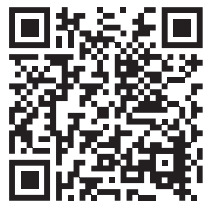


de la cirugía. Conclusión: El retraso de la cirugía no afecta la tasa de mortalidad después de una fractura de cadera en pacientes de edad avanzada con bajo ingreso económico.

Palabras clave: Fractura de cadera, tasa de mortalidad, índice de comorbilidad de Charlson.

\section{Introducción}

Las fracturas de cadera son reconocidas como la consecuencia más grave de la osteoporosis. Se asocian a complicaciones como dolor crónico, incapacidad para caminar, disminución de la calidad de vida o una muerte prematura. ${ }^{1,2}$ La mortalidad por fractura de cadera está relacionada con diversos factores de riesgo, incluyendo edad avanzada, género, comorbilidades, tipo de fractura y retraso de 48 horas o más para la cirugía. ${ }^{3,4,5,6}$ Sin embargo, otros autores sugieren que no existe una relación directa entre la mortalidad y las complicaciones o el retraso en la cirugía de fractura de cadera. ${ }^{7,8}$ La incidencia de fractura de cadera varía entre países y poblaciones. Los países industrializados, como los que se ubican en el norte de Europa y Estados Unidos de América (EUA), presentan las tasas más altas, mientras que América Latina y África tienen las más bajas. Los datos demográficos, las características étnicas y los factores ambientales se encuentran involucrados. ${ }^{1} \mathrm{El}$ riesgo de sufrir una fractura de cadera se incrementa en la quinta década de vida y se duplica cada 10 años. Más de $90 \%$ de las fracturas de cadera ocurren en pacientes mayores de 65 años. ${ }^{9,10,11,12}$ Se ha descrito que la tasa de mortalidad a un año incrementa entre 14 y 47\% después de una fractura de cadera, ${ }^{13,14,15,16}$ siendo mayor en hombres con comorbilidades asociadas. ${ }^{17,18}$ Asimismo, un paciente que requirió una reparación de fractura de cadera nunca regresa al nivel de actividad que tenía previo a la misma, a pesar de los avances en el tratamiento médico. Por lo tanto, las fracturas de cadera son una importante causa de morbimortalidad en los adultos mayores. ${ }^{18}$

Algunos autores han demostrado mayores tasas de supervivencia en los pacientes que son intervenidos quirúrgicamente en las primeras 48 horas. ${ }^{3,45,6}$ Sin embargo, otros estudios sugieren que un retraso de la cirugía de siete días no aumenta la mortalidad, mientras tanto, pueden ser atendidas otras comorbilidades. ${ }^{10,19}$ Las guías actuales sobre fracturas de cadera recomiendan realizar la cirugía dentro las primeras 24 horas en casos estables. ${ }^{20,21,22}$ Las principales causas de retraso en la cirugía son la falta de quirófano, personal médico o una enfermedad preexistente que deba estabilizarse antes el procedimiento quirúrgico. ${ }^{23}$ El objetivo de este estudio fue analizar la tasa de mortalidad y sus factores relacionados en pacientes de edad avanzada con fractura de cadera, en una población de bajos ingresos.
Keywords: Hip fracture, mortality rate, Charlson comorbidity index.

\section{Material y métodos}

Este estudio retrospectivo fue aprobado por el Comité de Ética de nuestra institución. Se incluyeron pacientes con reparación de fractura traumática de cadera en un período de cuatro años en un hospital universitario con un centro de entrenamiento en ortopedia. Los criterios de inclusión incluyeron: cualquier género, mayores de 60 años, fracturas traumáticas de cadera (cuello femoral, intertrocantérea o subtrocantérea) y registro médico completo. Se consideraron como criterios de exclusión las fracturas periprotésicas, fracturas patológicas y fracturas después de poliomielitis, así como la falta de datos completos o archivos perdidos. Los datos recolectados incluyeron: edad, género, fecha de admisión, tiempo de la cirugía después de la fractura, tiempo del procedimiento quirúrgico, tasa de transfusiones, complicaciones y fecha de egreso. El índice de comorbilidad de Charlson se calculó para todos los pacientes, este índice predice la mortalidad a un año de un paciente que cuenta con una variedad de comorbilidades, como enfermedades cardíacas, SIDA o cáncer (un total de 22 condiciones); es asignado un número del 1 al 6 que depende del riesgo de mortalidad de cada enfermedad. Los números son sumados para obtener un número total que permite predecir la mortalidad. ${ }^{24}$

Se realizó una entrevista telefónica al paciente o sus familiares para recabar información adicional, incluyendo causa o fecha de defunción. Los pacientes se distribuyeron en dos grupos, vivos o finados y los datos obtenidos se compararon entre ambos grupos.

\section{Análisis estadístico}

Se realizó una prueba de Kolmogorov-Smirnov para muestras independientes para analizar la distribución de datos $(\mathrm{p} \leq 0.05)$. Después se realizó una comparación entre grupos (vivos vs fallecidos); si la distribución era paramétrica, se empleó una prueba t-Student para variables independientes, o la prueba U de Mann-Whitney si no era paramétrica $(\mathrm{p} \leq 0.05)$. La prueba de $\chi^{2}$ o la prueba de Fisher fueron usadas para las variables nominales. Se utilizó el programa SPSS (IMB ${ }^{\circledR}$ SPSS $^{\circledR}$ Statistics versión 20 para Mac) para los análisis estadísticos.

\section{Resultados}

En el período de evaluación de cuatro años, 131 pacientes fueron atendidos por fractura traumática de cadera, 35 de ellos 
fueron excluidos para el propósito de este estudio. Un total de 96 pacientes con fracturas traumáticas de cadera fueron estudiados, de los cuales 26 (27\%) eran hombres y 70 (73\%) mujeres con un promedio de edad de $81 \pm 9$ años. Al final del seguimiento de cuatro años, 31 (32\%) casos fallecieron, de los cuales 16 (51.6\%) eventos fueron durante el primer año posterior a la cirugía, siete (22.5\%) durante el segundo año y ocho (25.8\%) después de dos años de evaluación. Los pacientes que sobrevivieron durante los cuatro años de evaluación mostraron mejores resultados de forma significativa en el índice de comorbilidades de Charlson (CCI) (Tabla 1).

El retraso de la cirugía y su impacto en la mortalidad fueron comparados entre ambos grupos. A pesar de un retraso promedio de $13 \pm 10$ días del tratamiento quirúrgico, no se observó diferencia estadísticamente significativa en mortalidad entre ambos grupos. En relación con el tiempo quirúrgico, tampoco se observó diferencia estadísticamente significativa entre ambos grupos (Tabla 1).

Además de la transfusión de hemocomponentes en 39 (68\%) de los hombres y 31 (54\%) de las mujeres, no se identificaron otras diferencias estadísticas en el género (Tabla 1).

La principal causa de muerte en 17 (54.8\%) casos fue desconocida, porque sus familiares no pudieron explicarlo. En cuatro (12.9\%) se presentó falla cardíaca; en cuatro (12.9\%) neumonía; en dos (6.25\%) casos accidente cerebrovascular; en dos (6.25\%) complicaciones de cáncer de pulmón y complicaciones de diabetes mellitus en otros dos (6.25\%).

En cuanto al tipo de fractura, la más frecuente fue la intertrocantérea con 55 (57\%) casos, y el procedimiento quirúrgico más común fue la reducción por maniobras cerradas y tornillo dinámico de cadera (DHS) en 52 (54\%) casos.

\section{Discusión}

Uno de los parámetros más importantes después de una cirugía por fractura de cadera es la tasa de mortalidad, prin- cipalmente en el primer año. En el siglo pasado, uno de cada dos pacientes con reparación de fractura de cadera fallecía. Sin embargo, esta tendencia ha evolucionado durante los últimos 50 años. Durante la década de los 80 se reportó una tasa de mortalidad de $24 \%$, en la década de los 90 fue de $23 \%$ y finalmente en 1999 se reportó en $21 \%,{ }^{22,23}$ Choi y colaboradores $^{25}$ reportaron en 2014 una tasa de mortalidad de $12 \%$ en el primer año posterior a la reparación de una fractura de cadera. Nuestro estudio identificó una tasa de mortalidad ligeramente más alta (16.4\%) durante el primer año de seguimiento; sin embargo, es una población de bajos ingresos sin cobertura de servicios de salud. No obstante, aún se encuentra en el rango reportado por Mundi y su grupo (14-36\%) en 2014. ${ }^{26}$

Para determinar si el retraso quirúrgico en la reparación de las fracturas de cadera tenía una relación significativa en la tasa de mortalidad, Desai y su equipo compararon pacientes diferidos contra no diferidos. En este estudio se encontró una diferencia estadísticamente significativa $(\mathrm{p} \leq 0.001)$ entre ambos grupos, presentando 93 horas contra 44 horas de cirugía retrasadas, respectivamente. ${ }^{27}$ Choi y colaboradores realizaron un estudio multicéntrico retrospectivo que incluyó 874 pacientes mayores de 65 años con fracturas de cadera. Fueron divididos según la fecha de la cirugía, encontrando una tasa de mortalidad de 9.9\% al año de la cirugía en el grupo de tratamiento temprano ( $\leq 3$ días) $(\mathrm{n}=162 ; 19 \%)$ comparado con $12.5 \%$ en el grupo de tratamiento tardío ( $\geq 3$ días) $(\mathrm{n}=712 ; 81 \%$ ), encontrando esta diferencia como no estadísticamente significativa. ${ }^{26}$ Los datos de nuestra investigación están de acuerdo con estos resultados, ya que no se observó diferencia en la mortalidad posterior a reparación de fractura de cadera según el tiempo de retraso en tiempo quirúrgico. A pesar de ser un centro de entrenamiento ortopédico, en el cual los residentes realizan la mayoría de los procedimientos quirúrgicos, no se observó diferencia entre ambos grupos en el tiempo de duración quirúrgica o en el tiempo de estancia intrahospitalaria. El retraso quirúrgico en nuestro centro se debe a que la mayoría de nuestra pobla-

\begin{tabular}{|c|c|c|c|c|}
\hline & $\begin{array}{l}\text { Vivos } \\
\mathrm{n}=65\end{array}$ & $\begin{array}{l}\text { Fallecidos } \\
\mathrm{n}=31\end{array}$ & $\begin{array}{l}\text { Total } \\
\mathrm{N}=96\end{array}$ & p \\
\hline Edad, años (rango) & $80 \pm 9(62-98)$ & $85 \pm 7(70-100)$ & $81 \pm 9(60-100)$ & $0.222 *$ \\
\hline \multicolumn{5}{|l|}{ Género, n (\%) } \\
\hline Masculino & $19(29)$ & 7 (23) & $26(27)$ & $0.625^{\ddagger}$ \\
\hline Femenino & $46(71)$ & $24(77)$ & $70(73)$ & \\
\hline $\begin{array}{l}\text { Índice de comorbilidad de Charlson } \\
\text { (rango) }\end{array}$ & $4.2 \pm 1.1(2-7)$ & $5.2 \pm 1.0(3-7)$ & $4.5 \pm 1.2(2-7)$ & $<0.001^{\S}$ \\
\hline Supervivencia a 10 años (rango) & $45.2 \pm 27.0(0.01-90.15)$ & $23.4 \pm 21.9(0.01-77.48)$ & $38.2 \pm 27.4(0.01-90.15)$ & $<0.001^{\S}$ \\
\hline Retraso en cirugía, días (rango) & $13 \pm 11(1-69)$ & $13 \pm 7(1-37)$ & $13 \pm 10(1-69)$ & $0.534^{\ddagger}$ \\
\hline Tiempo quirúrgico, horas (rango) & $3.38 \pm 1.06(2-7)$ & $3.3 \pm 1.60(2-7)$ & $3.3 \pm 1.0(2-7)$ & $0.619^{\S}$ \\
\hline Transfusión (unidad de sangre), n (\%) & $\begin{array}{c}36(55) \\
(0-4)\end{array}$ & $\begin{array}{c}21(68) \\
(0-4)\end{array}$ & $\begin{array}{c}57(59) \\
(0-4)\end{array}$ & $0.715^{\S}$ \\
\hline 1 & $16(44)$ & $13(42)$ & $29(51)$ & \\
\hline 2 & $16(44)$ & $7(23)$ & $23(40)$ & \\
\hline 3 & $3(7)$ & $0(0)$ & $3(5)$ & \\
\hline$>4$ & $1(2)$ & $1(3)$ & $2(4)$ & \\
\hline Estancia hospitalaria, días (rango) & $17 \pm 11(3-72)$ & $16 \pm 9(4-36)$ & $16 \pm 10(3-72)$ & $0.462^{\ddagger}$ \\
\hline
\end{tabular}


ción tiene bajos ingresos económicos. El paciente o su familia deben pagar el implante médico y la cirugía. En nuestro país, el ingreso diario aproximado es de 6.15 USD por día, y alrededor de 2,244 USD por año. ${ }^{28}$ En los últimos años, la tasa de desempleo en nuestro país ha disminuido, aunque se encuentra alrededor de 5\%. La brecha social entre la población de bajos ingresos y las clases sociales más altas es muy significativa, lo que hace que la población de mayor ingreso gane 20 veces más que $10 \%$ de la población más pobre. Mientras tanto, en otros países el promedio se incrementa ocho veces. A pesar de las mejoras en los sistemas de salud con medidas preventivas, tales como la nutrición y la detección de enfermedades, los indicadores de salud siguen siendo preocupantes. Asimismo, los desembolsos personales y los costos administrativos indican ineficiencias y accesos desiguales a la población. ${ }^{29}$

El índice de comorbilidades de Charlson evalúa la esperanza de vida a 10 años, a través de un sistema de evaluación con puntajes establecidos sobre la edad y comorbilidades del sujeto. ${ }^{24}$ En un estudio multicéntrico de Choi y su equipo ${ }^{25}$ 719 (82\%) pacientes tenían un ICC de 0 a 1; en contraste con nuestra población antes del evento de la fractura, en la cual más de 95\% tuvieron CCI de dos o más. Esto puede explicar el incremento de la tasa de mortalidad obtenida.

Las limitaciones de este estudio incluyen un bajo número de pacientes analizados; una muestra más amplia nos daría una mejor perspectiva de la enfermedad. Además, no se encontraron registrados en el expediente los motivos para retrasar las cirugías. Esto hubiera sido útil para identificar problemas y crear estrategias para solucionarlos.

\section{Conclusión}

A pesar de ser un instituto con recursos económicos limitados, el retraso en la cirugía y la duración del procedimiento quirúrgico no incrementan la tasa de mortalidad posterior al tratamiento de fracturas de cadera en una población de adultos mayores de bajo nivel socioeconómico.

Referencias

1. Dhanwal DK, Dennison EM, Harvey NC, Cooper C. Epidemiology of hip fracture: Worldwide geographic variation. Indian J Orthop. 2011; 45(1): 15-22.

2. Quevedo I, Ormeño JC, Weissglas B, Opazo C. Epidemiology and direct medical cost of osteoporotic hip fracture in Chile. J Osteoporos. 2020: 2020: 5360467.

3. Sheehan KJ, Sobolev B, Guy P. Mortality by timing of hip fracture surgery: factors and relationships at play. J Bone Joint Surg Am. 2017; 99(20): e106.

4. Dailiana Z, Papakostidou I, Varitimidis S, Michalitsis SG, Veloni A, Malizos KN. Surgical treatment of hip fractures: factors influencing mortality. Hippokratia. 2013; 17(3): 252-7.

5. Meessen JM, Pisani S, Gambino ML, Bonarrigo D, van Schoor NM, Fozzato S, et al. Assessment of mortality risk in elderly patients after proximal femoral fracture. Orthopedics. 2014; 37(2): e194-200.

6. Trpeski S, Kaftandziev I, Kjaev A. The effects of time-to-surgery on mortality in elderly patients following hip fractures. Prilozi. 2013; 34(2): 115-21.

7. Etxebarria-Foronda I, Mar J, Arrospide A, Ruiz de Eguino J. Cost and mortality associated to the surgical delay of patients with a hip fracture. Spain. Rev Esp Salud Publica. 2013; 87(6): 639-49.
8. Switzer JA, Bennett RE, Wright DM, Vang S, Anderson CP, Vlasak AJ, Gammon SR. Surgical time of day does not affect outcome following hip fracture fixation. Geriatr Orthop Surg Rehabil. 2013; 4(4): 109-16.

9. Walter LC, Lui LY, Eng C, Covinsky KE. Risk of hip fracture in disabled community-living older adults. J Am Geriatr Soc. 2003; 51(1): 50-5.

10. Zuckerman JD. Hip fracture. N Engl J Med. 1996; 334(23): 1519-25.

11. Dennison E, Cooper C. Epidemiology of osteoporotic fractures. Horm Res. 2000; 54[Suppl 1]: 58-63.

12. Panula J, Pihlajamäki H, Mattila VM, Jaatinen P, Vahlberg T, Aarnio P, Sirkka-Liisa K. Mortality and cause of death in hip fracture patients aged 65 or older: a population-based study. BMC Musculoskelet Disord. 2011; 12: 105.

13. Forster MC, Calthorpe D. Mortality following surgery for proximal femoral fractures in centenarians. Injury. 2000; 31(7): 537-9.

14. Herrera A, Domingo LJ, Calvo A, Martinez A, Cuenca J. A comparative study of trochanteric fractures treated with the Gamma nail or the proximal femoral nail. Int Orthop. 2002; 26(6): 365-9.

15. González-Rozas M, Pérez-Castrillón JL, González-Sagrado M, RuizMambrilla M, García-Alonso M. Risk of mortality and predisposing factors after osteoporotic hip fracture: a one-year follow-up study. Aging Clin Exp Res. 2012; 24(2): 181-7.

16. Gronskag AB, Romundstad P, Forsmo S, Langhammer A, Schei B. Excess mortality after hip fracture among elderly women in Norway: the HUNT study. Osteoporos Int. 2012; 23(6): 1807-11.

17. Jamal Sepah Y, Umer M, Khan A, Ullah Khan Niazi A. Functional outcome, mortality and in-hospital complications of operative treatment in elderly patients with hip fractures in the developing world. Int Orthop. 2010; 34(3): 431-35.

18. Abrahamsen B, van Staa T, Ariely R, Olson M, Cooper C. Excess mortality following hip fracture: a systematic epidemiological review. Osteoporos Int. 2009; 20(10): 1633-50.

19. Lawrence VA, Hilsenbeck SG, Noveck H, Poses RM, Carson JL. Medical complications and outcomes after hip fracture repair. Arch Intern Med. 2002; 162(18): 2053-57.

20. Doruk H, Mas MR, Yildiz C, Sonmez A, Kyrdemir V. The effect of the timing of hip fracture surgery on the activity of daily living and mortality in elderly. Arch Gerontol Geriatr. 2004; 39(2): 179-85.

21. Egol KA, Strauss EJ. Perioperative considerations in geriatric patients with hip fracture: what is the evidence. J Orthop Trauma. 2009; 23(6): 386-94.

22. Gdalevich M, Cohen D, Yosef D. Morbidity and mortality after hip fracture: the impact of operative delay. Arch Orthop Trauma Surg. 2004; 124(5): 334-40.

23. Simunovic N, Devereaux PJ, Bhandari M. Surgery for hip fractures: Does surgical delay affect outcomes? Indian J Orthop. 2011; 45(1): 27-32.

24. Charlson ME, Pompei P, Ales KL, MacKenzie CR. A new method of classifying prognostic comorbidity in longitudinal studies: development and validation. J Chronic Dis. 1987; 40(5): 373-83.

25. Choi HJ, Kim E, Shin YJ, Choi BY, Kim YH, Lim TH. The timing of surgery and mortality in elderly hip fractures. A retrospective, multicentric cohort study. Indian J Orthop. 2014; 48(6): 599-604.

26. Mundi S, Pindiprolu B, Simunovic N, Bhandari M. Similar mortality rates in hip fracture patients over the past 31 years. A systematic review of RCTs. Acta Orthop. 2014; 85(1): 54-9.

27. Desai SJ, Patel J, Abdo H, Lawendy AR, Sanders D. A comparison of surgical delays in directly admitted versus transferred patients with hip fractures: opportunities for improvement? Can J Surg. 2014; 57(1): 40-3.

28. Gobierno de México. https://www.gob.mx/stps/prensa/con-aumentode-20-al-salario-minimo-para-2020-mexico-tiene-las-bases-paracrecer-afirma-presidente-lopez-obrador-230226?idiom=es

29. OECD (Organization for Economic Co-operation and Development) [Data accessed 24/04/2020] Available in: www.oecd.org/eco/surveys/ economic-survey-mexico.htm

Conflicto de intereses: Todos los autores declaran no tener conflicto de intereses con la realización y publicación del presente trabajo. 